Springer $\underline{\text { Springer }}$ Springer

\section{Schulterendoprothese wegen Omarthrose erzielt gute Resultate}

Wenn alles optimal läuft, verschafft der Totalersatz eines arthrotischen Schultergelenks den Patienten eine eindrucksvolle Verbesserung der Schmerzen wie auch der Gelenkfunktion.

E twa 3.000 Schulter-Totalenedopro- thesen (TEPs) werden in Deutschland pro Jahr implantiert. Welchen Gewinn sich die Patienten davon erwarten dürfen, wurde nun mithilfe einer Metaanalyse untersucht. Ausgewertet wurden 20 Studien, überwiegend Fallserien, in denen 1.576 Schultergelenke in großen Zentren von erfahrenen Chirurgen ersetzt worden waren. Die Patienten waren durchschnittlich 66 Jahre alt. Hauptindikation für den Gelenkersatz war eine primäre Omarthrose, die Beobachtungsdauer betrug im Schnitt 3,7 Jahre.

Vor der Operation bezifferten die Patienten ihre Schmerzen auf einer visuellen Analogskala von 0-10 im Mittel auf 7,2; postoperativ sank der Wert auf 1,4. Die Schulterfunktion verbesserte sich ebenfalls erheblich. So stieg beispielsweise die im American Shoulder and Elbow Surgeons (ASES)-Schulter-Score erreichte Punktzahl von 34,8 auf 82,5 . Als klinisch relevant gilt bereits eine Differenz von 6,4 Punkten. Im Constant Score, der wie der ASES eine Kombination aus Schmerz, Schulterbeweglichkeit, Kraft und Bewältigung von Alltagsaktivitäten bewertet, zeigte sich ebenfalls eine hochsignifikante Verbesserung von 31,7 auf 69,8 Punkte. Im Simple Shoulder Test konnten die Patienten von zwölf Schulteraktivitäten, etwa Heben eines Gewichts in Schulterhöhe, statt wie vorher 3,1 nach der TEP-Implantation 9,6 Übungen erfolgreich ausführen. Darüber hinaus nahm auch die gesundheitsbezogene Lebensqualität Patienten $\mathrm{zu}$, was an einem signifikanten Anstieg von 35, auf 42,9 im Summenscore der physischen Komponenten des SF-36 ablesbar war.

Fazit: In allen Studien dieser Übersichtsarbeit erwies sich die totale Schulterarthroplastik bei Omarthrose als hochwirksame Intervention in Bezug auf
Funktion und Schmerzen sowie die gesundheitsbezogene Lebensqualität. Einschränkend ist allerdings festzustellen, dass die zugrundliegenden Daten fast ausschließlich aus Fallserien stammen.

Dr. Beate Schumacher

Carter MJ et al. Impact of Total Shoulder Arthroplasty on Generic and Shoulder-Specific HealthRelated Quality-of-Life Measures. J Bone Joint Surg AN 2012;94:e127(1-9)

Kommentar: In dieser Metaanalyse wurde schön herausgearbeitet, dass bei einem arthrotischen Schultergelenk eine deutliche Verbesserung durch die Implantation einer Totalendoprothese erzielt werden kann. Die guten Ergebnisse entsprechen dabei auch unseren Erfahrungen. Allerdings ist zu beachten, dass es sich in der Analyse fast ausschließlich um primäre Schulterprothesen ohne wesentliche Begleitpathologien handelt. Veränderungen an der Rotatorenmanschette und am Glenoid haben jedoch einen erheblichen Einfluss auf die postoperative Funktion und die Standzeit einer Schulterprothese. Auch handelt es sich in der Metanalyse um Frühergebnisse nach durchschnittlich 3,7 Jahren. Langzeitergebnisse sowie Resultate nach Revisionseingriffen sind bisher nur in begrenztem Umfang vorhanden. Eine sorgfältige Indikationsstellung unter Berücksichtigung aller Faktoren ist daher auch bei Schulterprothesen essenziell, um nicht nur kurzfristig, sondern auch langfristig gute Ergebnisse zu erzielen.

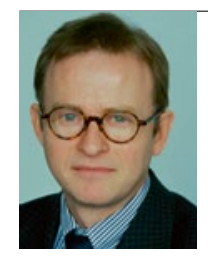

Prof. Volkmar Jansson Orthopädische Klinik und Poliklinik der LMU Klinikum Großhadern Marchioninistr. 15 D-81377 München
Q Springer $\underline{\text { Springer }}$ Springe

Springer $\underline{\text { Springer }}$ Springer

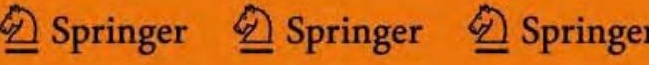

Springer $\underline{\text { Springer }} \underline{\underline{\text { Springer }}}$

$\underline{\text { Springer }}$ Springer $\underline{\underline{\text { Springe }}}$

Springer $\underline{\text { Springer }}$ Springer

Springer Springer Springer

Springer $\underline{\text { Springer }}$ Springer

Q Springer $\underline{\text { Springer }}$ Springe

Springer Springer Springer

QSpringer $\underline{\text { Springer }} \underline{\underline{\text { Springel }}}$

Springer 2 Springer 2 Springer

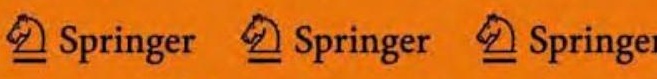

Springer $\underline{\text { Springer }} \underline{\underline{\text { Springer }}}$

Q Springer $\underline{\text { Springer }}$ Springe

Springer Springer $\underline{\text { Springer }}$

QSpringer $\underline{\text { Springer }} \underline{\underline{\text { Springer }}}$

Q Springer 2 Springer 2 Springer

QSpringer $\underline{\text { Springer }}$ Springer

Springer $\triangleq$ Springer $\cong$ Springer

Q Springer Springer Springer

Q Springer 2 Springer 2 Springer

政pringer Springer Springer

Springer Springer Springer

은 Springer Springer $\underline{\underline{\text { Springe }}}$

Springer $\underline{\text { Springer }}$ Springer

Q Springer Springer Springer

Springer $\underline{\text { Springer }}$ Springer

QSpringer $\triangleq$ Springer $\triangleq$ Springe

Springer $\underline{\text { Springer }}$ Springer

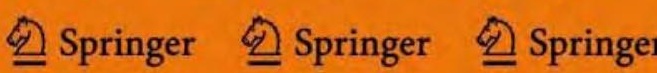

Springer Springer Springer

Q Springer Springer

ORTHOPÄDIE \& RHEUMA 2013; 16 (1) 